\title{
THE INTERACTION OF OBLIQUE WAVES WITH A PARTIALLY IMMERSED WAVE ABSORBING BREAKWATER
}

\author{
Yong $\mathrm{Liu}^{1}$ and Yucheng $\mathrm{Li}^{2}$
}

\begin{abstract}
By considering obliquely incident waves, the hydrodynamic performance of a partially immersed wave absorbing breakwater is examined in this study. The breakwater consists of a perforated front barrier and a solid rear barrier. The two barriers are both partially immersed with the same draft. An analytical solution based on the linear potential theory is developed to calculate the reflection and transmission coefficients of the breakwater and the wave forces acting on the barriers. Some useful results are presented according to numerical examples. The present solution may be used at a preliminary design stage in practical engineering.
\end{abstract}

Keywords: partially immersed barrier; perforated barrier; oblique wave; analytical solution

\section{INTRODUCTION}

Various perforated or porous breakwaters have been widely used in coastal engineering due to their powerful abilities of dissipating wave energy from open sea. The dissipation of wave energy can lead to lower reflection coefficients. This is significant to suppress the seabed scouring in front of the breakwater. The lower reflection is also helpful to the safe navigation and berth of vessels near the structures. So studying the hydrodynamic performance of perforated or porous breakwaters, which may also be called as wave absorbing breakwater, is important for practical engineering.

A typical and often used wave absorbing breakwater proposed by Jarlan (1961) includes a perforated front wall, a solid rear wall and a wave absorbing chamber between them. The wave absorbing performance of this type of breakwater is mainly determined by the relative wave chamber width $B / L$, where $B$ is the wave chamber width and $L$ is the incident wavelength. According to Chwang and Dong (1984) and Fugazza and Natale (1992), the original Jarlan-type breakwater will attain a minimum reflection coefficient at $B / L=0.25$ in theory, if ignoring the inertial effect of the perforated front wall. More studies on the Jarlan-type breakwater can be found in many published literatures (e.g., Tanimoto and Yoshimoto, 1982; Bennett et al., 1992, Suh et al., 2006, Li, 2007).

It is noted that the traditional wave absorbing breakwaters are bottom-standing. Thus the free water circulation between the open sea and the shelter region fails. An alternative wave absorbing breakwater is partially immersed perforated structures (e.g., Cox et al., 1998; Brossard et al., 2003). For this concept, the wave absorbing structure is fixed by plies and extends from above the sea surface to some distance above the seabed. Then the under gaps allow the seawater circulation, the sediment transport and the fish passage. This can prevent the deterioration of coastal marine environment. The partially immersed wave absorbing breakwater can also dissipate the incident wave energy effectively, and thus may lead to simultaneous lower reflection and transmission coefficients. This is rather significant for practical engineering. However, analytical studies on the hydrodynamic performance of partially immersed wave absorbing breakwater have been scarcely referred so far. This is just the main objective of this paper. In the next section, an analytical solution for the interaction of oblique waves with partially immersed wave absorbing breakwater is developed. In section 3, the analytical solution is validated by previous predictions for limiting cases and previous experimental data. In section 4, numerical examples are presented to examine the hydrodynamic performance of the breakwater. Finally, the main conclusion of this study is drawn.

\section{MATHEMATICAL FORMULATION AND SOLUTIONS}

As shown in Fig. 1, we consider the interaction of obliquely incident waves with a partially immersed wave absorbing breakwater. The breakwater includes a perforated front barrier and a solid rear barrier. The 3D Cartesian-coordinate system is adopted to describe the present problem. The origin is located in the intersection of the still water level and the midline of two barriers. The $x$-axis is in direction of wave propagation, the $y$-axis lies along the length direction of the breakwater, and the $z$ axis points vertically upwards. The two barriers have the same draft denoted by $a$. The space between the two barriers is $B$. The water depth is $d$. The height and wavelength of incident wave are $H$ and $L$,

\footnotetext{
${ }^{1}$ College of Engineering, Ocean University of China, No. 238 Song Ling Road, Qingdao, Shandong, 266100, China

${ }^{2}$ State Key Laboratory of Coastal and Offshore Engineering, Dalian University of Technology, No. 2 Ling Gong Road, Dalian, Liaoning, 116024, China
} 
respectively. The included angle between the incident wave propagation direction and the positive $x$ direction is $\beta$. To obtain the analytical solution, we divide the whole fluid domain into three regions: region 1, the fluid domain in front of the front barrier; region 2, the fluid domain between the two barriers; and region 3, the fluid domain behind the rear barrier. We note that the thicknesses of the two barriers are both very small in comparison with the incident wavelength. So they are both treated as zero for the scattering of water waves by the breakwater.
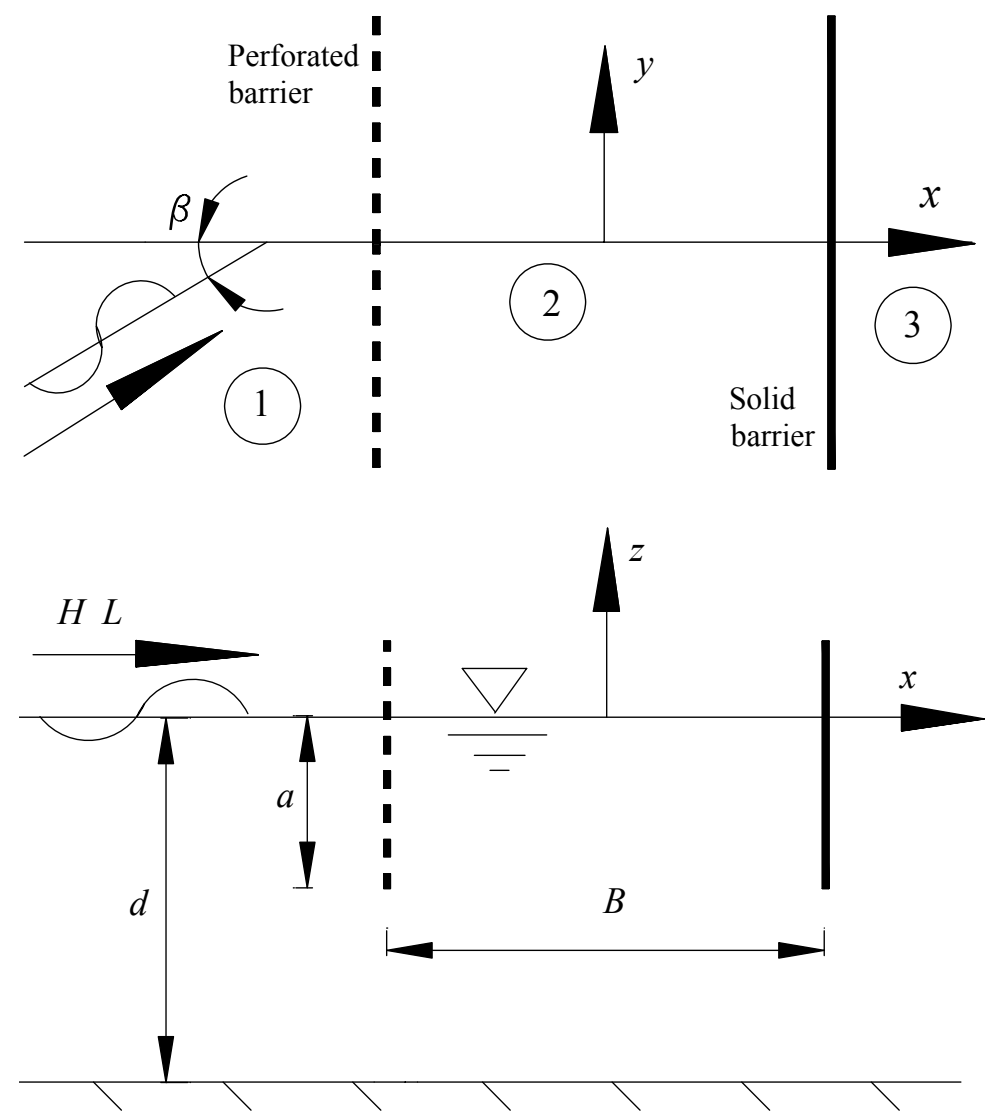

Figure 1. Definition sketch for the breakwater: the upper is bird view and the under is side view.

Considering linear potential theory and harmonic waves with an angular frequency $\omega$, we can write the fluid velocity as

$$
\Phi(x, y, z, t)=\operatorname{Re}\left[\phi(x, z) \mathrm{e}^{\mathrm{i} k_{0 y} y} \mathrm{e}^{-\mathrm{i} \omega t}\right]
$$

where $g$ is gravitational acceleration, $k_{0 y}$ is the component of incident wave number $k_{0}$ in the $y$ direction, and $t$ is the time. Then our main objective is to obtain the time-independent velocity potential $\phi$. The spatial potential in each sub region satisfies the modified Helmholtz equation:

$$
\frac{\partial^{2} \phi_{j}}{\partial x^{2}}+\frac{\partial^{2} \phi_{j}}{\partial z^{2}}-k_{0 y}^{2} \phi_{j}=0, j=1,2,3
$$

where the subscript $j$ denotes variables in the sub-region $j$. The spatial potentials also satisfy the linear free surface condition and boundary condition on the seabed:

$$
\begin{aligned}
& \frac{\partial \phi_{j}}{\partial z}=\frac{\omega^{2}}{g} \phi_{j}, z=0, j=1,2,3 \\
& \frac{\partial \phi_{j}}{\partial z}=0, z=-d, j=1,2,3
\end{aligned}
$$

In addition, the reflected and transmitted waves must be outgoing in the far fields.

By the separation of variables, the velocity potentials satisfying the modified Helmholtz equation and the above boundary conditions can be written as: 


$$
\begin{aligned}
& \phi_{1}=-\frac{\mathrm{i} g H}{2 \omega}\left[\mathrm{e}^{-\alpha_{0}(x+B / 2)} Z_{0}(z)+\sum_{n=0}^{\infty} R_{n} \mathrm{e}^{\alpha_{n}(x+B / 2)} Z_{n}(z)\right] \\
& \phi_{2}=-\frac{\mathrm{i} g H}{2 \omega}\left[\sum_{n=0}^{\infty} A_{n} \mathrm{e}^{-\alpha_{n}(x+B / 2)} Z_{n}(z)+\sum_{n=0}^{\infty} C_{n} \mathrm{e}^{\alpha_{n}(x-B / 2)} Z_{n}(z)\right] \\
& \phi_{3}=-\frac{\mathrm{i} g H}{2 \omega} \sum_{n=0}^{\infty} T_{n} \mathrm{e}^{-\alpha_{n}(x-B / 2)} Z_{n}(z)
\end{aligned}
$$

where, $\alpha_{0}=-\mathrm{i} k_{0 x}, \alpha_{n}=\sqrt{{k_{n}{ }^{2}+k_{0 y}{ }^{2}}_{j}} ; k_{0 x}=k_{0} \cos \beta, k_{0 y}=k_{0} \sin \beta ; R_{n}, A_{n}, C_{n}$ and $T_{n}$ are unknown complex expansions coefficients, and $Z_{n}(z)$ are depth-dependent functions as follows:

$$
Z_{n}(z)=\left\{\begin{array}{l}
\cosh k_{0}(z+d) / \cosh k_{0} d, n=0 \\
\cos k_{n}(z+d) / \cos k_{n} d, n=1,2, \ldots
\end{array}\right.
$$

The wave numbers $k_{n}$ are the positive roots of the following dispersion relations:

$$
\omega^{2}=g k_{0} \tanh \left(k_{0} d\right)=-\tan \left(k_{n} d\right), n=1,2, \ldots
$$

To obtain the four sets of unknown coefficients in Eqs. (5) - (7), we consider the following matching conditions between different sub-regions:

$$
\begin{aligned}
& \frac{\partial \phi_{1}}{\partial x}=\frac{\partial \phi_{2}}{\partial x}, x=-\frac{B}{2} \\
& \frac{\partial \phi_{3}}{\partial x}=\frac{\partial \phi_{2}}{\partial x}, x=\frac{B}{2} \\
& \frac{\partial \phi_{1}}{\partial x}=\mathrm{i} k_{0} G\left(\phi_{1}-\phi_{2}\right),-a \leq z \leq 0, x=-\frac{B}{2} \\
& \phi_{1}=\phi_{2},-d \leq z \leq-a, x=-\frac{B}{2} \\
& \frac{\partial \phi_{3}}{\partial x}=0,-a \leq z \leq 0, x=\frac{B}{2} \\
& \phi_{3}=\phi_{2},-d \leq z \leq-a, x=\frac{B}{2}
\end{aligned}
$$

where $G$ is the porous effect parameter defined as follows (Yu, 1995):

$$
G=\frac{\varepsilon}{k_{0} \delta(f-\mathrm{i} s)}
$$

in which, $\varepsilon, f$ and $s$ are the porosity, the linearized resistance coefficient and the inertial effect coefficient of the perforated barrier (Sollitt and Cross, 1972); and $\delta$ is the thickness of the barrier. We note that the thickness of the barrier is considered when calculating the porous effect parameter. We also note that when $|G|=0$, the perforated barrier reduces to a solid barrier; while for $|G| \rightarrow+\infty$, the barrier becomes entirely transparent. Moreover, the Eq. (12a) is the porous plate boundary condition $(\mathrm{Yu}, 1995)$. It denotes that the normal fluid velocity passing through the perforated barrier is linearly proportional to the pressure difference between the two sides of the barrier.

Inserting Eqs. (5) - (7) into Eqs. (10) and (11) and using the orthogonal relation $\int_{-d}^{0} Z_{m}(z) Z_{n}(z) \mathrm{d} z=0(m \neq n)$, gives:

$$
\begin{aligned}
& R_{n}=\delta_{n 0}-A_{n}+\mathrm{e}^{-\alpha_{n} B} C_{n} \\
& T_{n}=\mathrm{e}^{-\alpha_{n} B} A_{n}-C_{n}
\end{aligned}
$$

where $\delta_{n m}=1$ at $n=m$ and $\delta_{n m}=0$ at $n \neq m$. Inserting Eqs. (5) - (7) and Eqs. (15) and (16) into the matching conditions, Eqs. (12) and (13), gives two set of double series with the unknown coefficients of $A_{n}$ and $C_{n}$. By using a least square method (Dalrymple et al., 1990) and truncating $n$ to $N$, the two double series are transformed into two sets of linear equations. Solving the linear equations gives the unknown coefficients. Then various hydrodynamic parameters of engineering interest can be obtained.

The real reflection and transmission coefficients, $R$ and $T$, are calculated by

$$
R=\left|R_{0}\right|
$$




$$
T=\left|T_{0}\right|
$$

The spatial dynamic pressure at any point in the fluid domain can be calculated by using the linear Bernoulli equation:

$$
P(x, y, z, t)=\operatorname{Re}\left[p(x, z) \mathrm{e}^{\mathrm{i} k_{0 y} y} \mathrm{e}^{-\mathrm{i} \omega t}\right]=\operatorname{Re}\left[\mathrm{i} \rho \omega \phi(x, z) \mathrm{e}^{\mathrm{i} k_{0 y} y} \mathrm{e}^{-\mathrm{i} \omega t}\right](19)
$$

where $\rho$ is the fluid density. We note that for oblique waves, the dynamic pressure acting on the breakwater varies periodically along the $y$-direction. So we only consider the wave forces acting on the cross section of the barriers. Here the wave forces are normalized by $\rho g H d$. Then the dimensionless wave forces acting on the front and rear barriers can be calculated, respectively, by

$$
\begin{aligned}
& F_{f}=\left.\frac{\mathrm{i} \omega}{g H d} \int_{-a}^{0}\left(\phi_{1}-\phi_{2}\right)\right|_{x=-B / 2, y=0} \mathrm{~d} z \\
& F_{r}=\left.\frac{\mathrm{i} \omega}{g H d} \int_{-a}^{0}\left(\phi_{2}-\phi_{3}\right)\right|_{x=B / 2, y=0} \mathrm{~d} z
\end{aligned}
$$

It should be mentioned that in the following calculations, a large truncated value of $N=400$ is used due to the singularity in flow at the tips of the barriers. At this time, the results are sufficiently accurate for engineering purposes.

\section{VALIDATIONS}

The present solution was validated by compared the calculated results with previous analytical results (Porter, 1995; Das et al., 1997) and previous experimental data (Cox et al., 1998).

When $|G|$ approaches infinity, the perforated front barrier vanishes and the present breakwater reduces to a single partially immersed solid barrier. At this time, the present calculated reflection and transmission coefficients were compared with the corresponding results of Porter (1995), as shown in Fig. 2. The calculating conditions are: $a / d=0.5, G=+\infty$ and $k_{0} d=2.0$. The results of Porter (1995) were obtained by a multi-term Galerkin approximation. Their results are extreme accurate. It can be seen from Fig. 2 that the agreement between present prediction and Porter (1995) is very well. When $|G|$ approaches zero, the perforated front barrier becomes a solid barrier and the present breakwater reduces to double partially immersed solid barriers. At this time, the present calculated reflection coefficient was compared with the corresponding result of Das et al. (1997), as shown in Fig. 3. Here the calculating conditions are: $a / d=0.6, G=0.0, B / d=2.0$ and $\beta=30^{\circ}$. It is evident from Fig. 3 that the agreement between the two different results is very well.

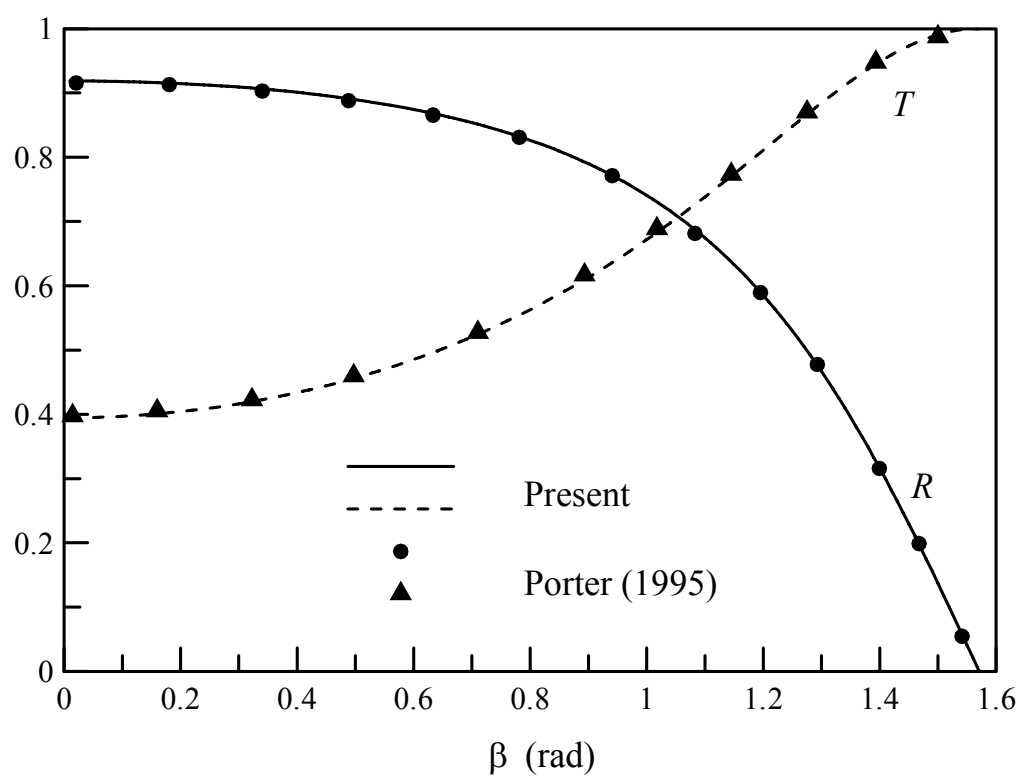

Figure 2. Comparison between present results and predictions of Porter (1995): $a / d=0.5, G=+\infty$ and $\boldsymbol{k}_{0} d=$ 2.0. 


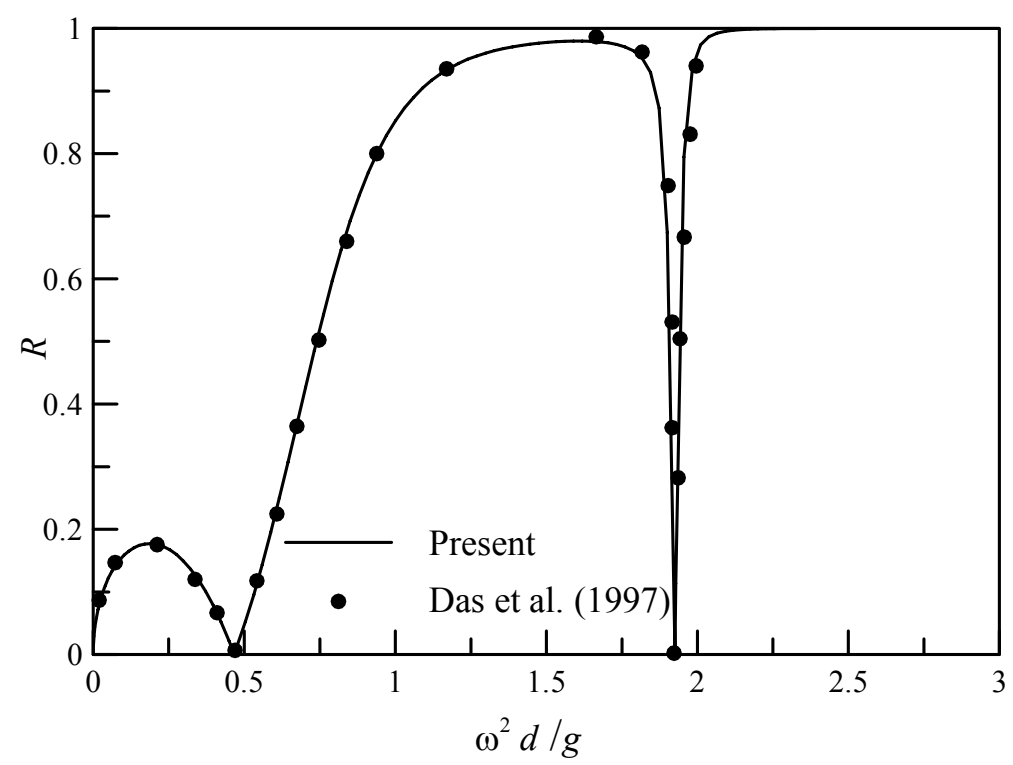

Figure 3. Comparison between present results and predictions of Das et al. (1997): $a / d=0.6, G=0.0, B / d=$ 2.0 and $\beta=30^{\circ}$.

For normally incident waves, Cox et al. (1998) have obtained a series of experimental data for the reflection coefficient of the partially immersed wave absorbing breakwater. The present predictions of the reflection coefficient were compared with the experimental results of Cox et al. (1998), as shown in Fig. 4. Here the porous effect parameter $G$ of the front barrier was calculated by Eq. (14). For calculating $G, \varepsilon=20 \%$ was adopted as used in the tests, $s=1$ was adopted as usual, and the resistance coefficient $f$ was estimated by a simply empirical formula of Li et al. (2006): $f=-3338.7(\delta / d)^{2}+82.769(\delta / d)+8.711(0.0094 \leq \delta / d \leq 0.05)$. The value of the dimensionless barrier thickness $\delta / d$ (barrier thickness / water depth) was not given in Cox et al. (1998). A reasonable value of $\delta / d=0.02$ was adopted. It can be seen from Fig. 4 that the present results agree reasonable with the experimental data of Cox et al. (1998).

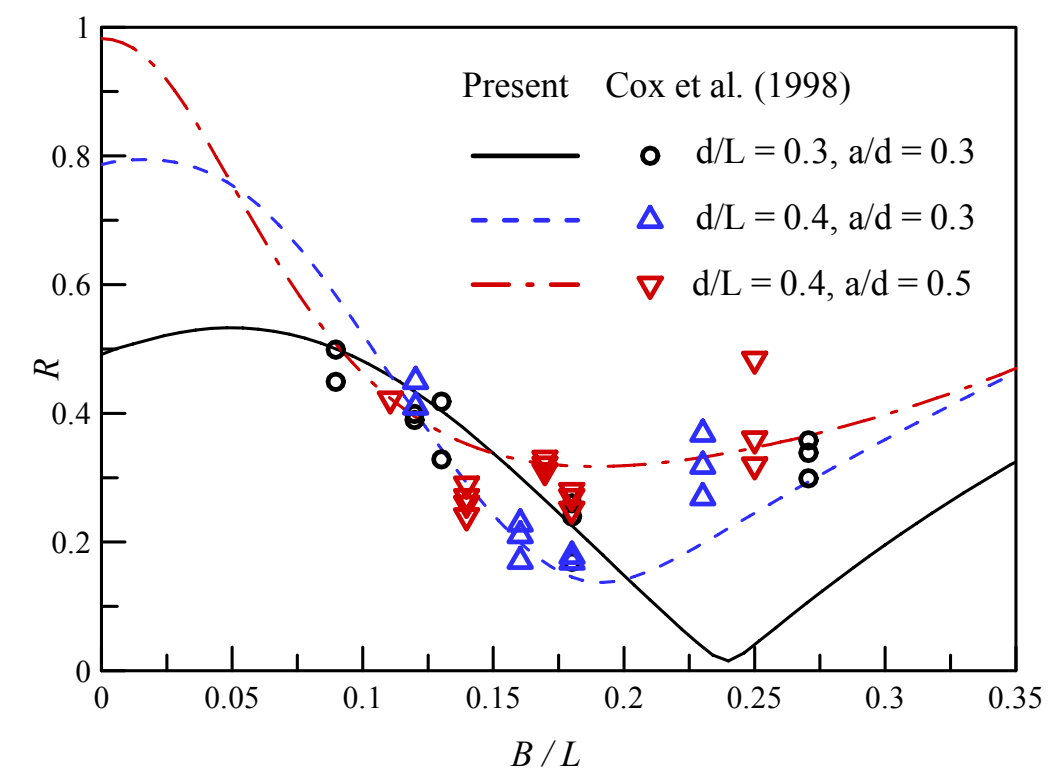

Figure 4. Comparison between present results and experimental data of Cox et al. (1998): $\beta=0^{\circ}$ and $\varepsilon=20 \%$. 


\section{RESULTS WITH DISCUSSION}

Figs. 5 and 6 show the effects of the relative space $B / L$ between the two barriers on $R, T, F_{f}$ and $F_{r}$. Here the calculating conditions are: $k_{0} d=2.0, a / d=0.4, \beta=30^{\circ}$ and $G=0$ in Fig. 5 and $G=2.0$ in Fig. 6. It can be seen from Fig. 5 that for the double solid barriers, the reflection coefficient may attain a very small value (even zero) with the increasing value of $B / L$. But the transmission coefficient corresponding to the smaller reflection coefficient is very large. So the smaller reflection coefficient is not significant in practice. For the present wave absorbing structure, it is satisfactory to find in Fig. 6 that the reflection and transmission coefficients can simultaneously attain lower values. This is due to the wave energy loss caused by the perforated front barrier. At this time, the wave force coefficients $F_{f}$ and $F_{r}$ are also small. This is a mainly merit of the present wave absorbing breakwater.

The effects of the angle of incident waves on $R, T, F_{f}$ and $F_{r}$ are shown in Figs. 7 and 8 . Here the calculating conditions are: $k_{0} d=2.0, G=0.3, a / d=0.4$ and $B / L=0.2$ in Fig. 7 and $B / L=0.6$ in Fig. 8 . It can be seen from these two figures that the variations of $R, T, F_{f}$ and $F_{r}$ versus $\beta$ is relevant to the value of $B / L$. Generally, a trough can appear in the curve of the reflection coefficient with the increasing value of $\beta$. When the angle of incident waves tends towards $90^{\circ}$, the transmission coefficient approaches unity and the reflection coefficient and the dimensionless wave forces all approach zero. By considering all the four hydrodynamic parameters, the partially immersed wave absorbing breakwater works better at a moderate angle of incident waves.

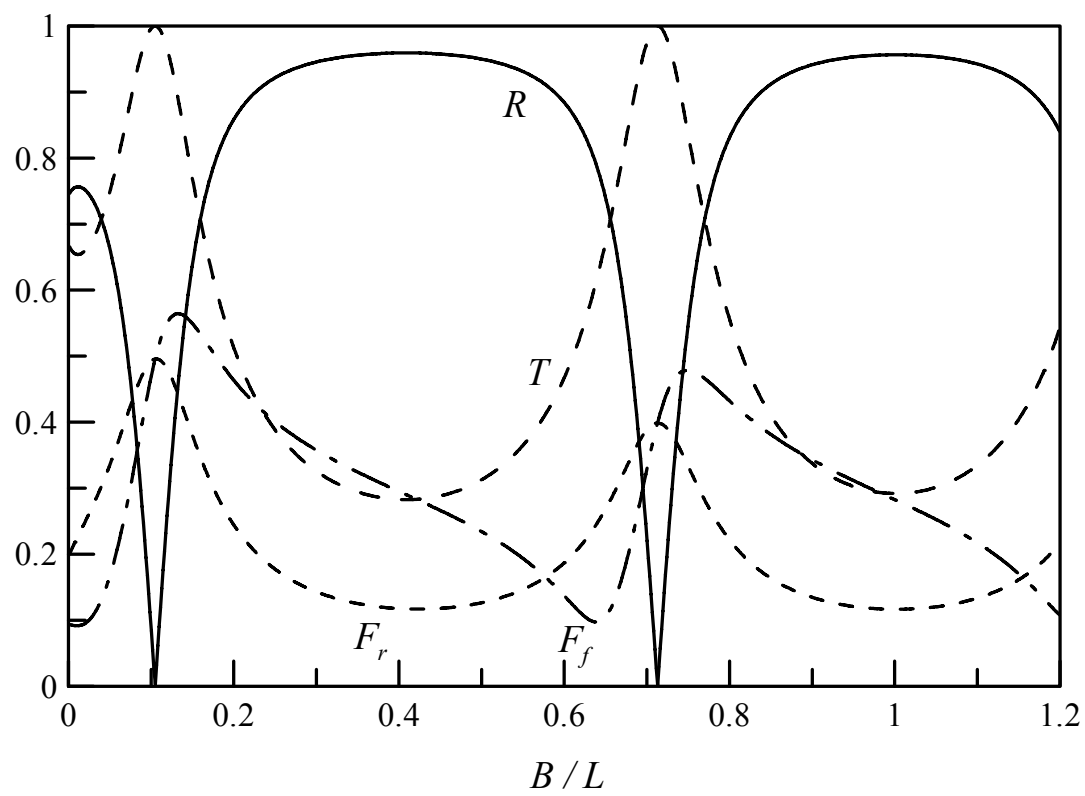

Figure 5. Effects of $B / L$ on $R, T, F_{f}$ and $F_{r}: k_{0} d=2.0, G=0.0$, ald $=0.4$ and $\beta=30^{\circ}$. 


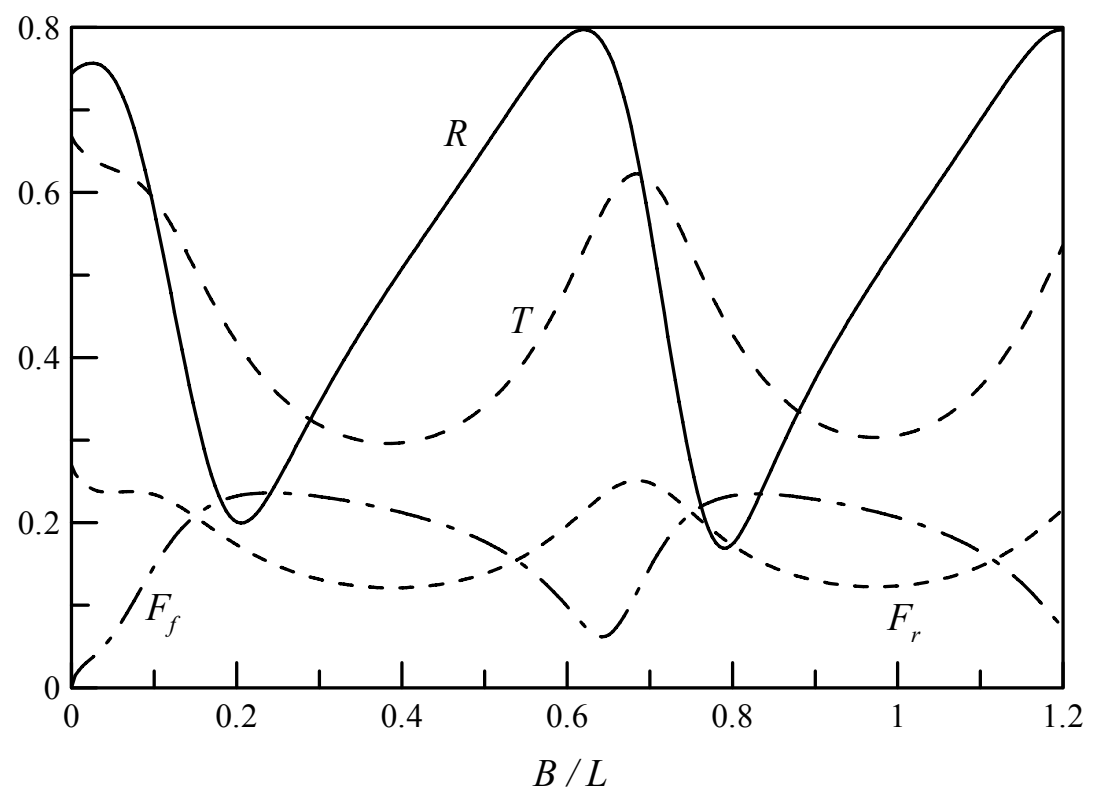

Figure 6. Effects of $B / L$ on $R, T, F_{f}$ and $F_{r}: k_{0} d=2.0, G=0.3, a / d=0.4$ and $\beta=30^{\circ}$.

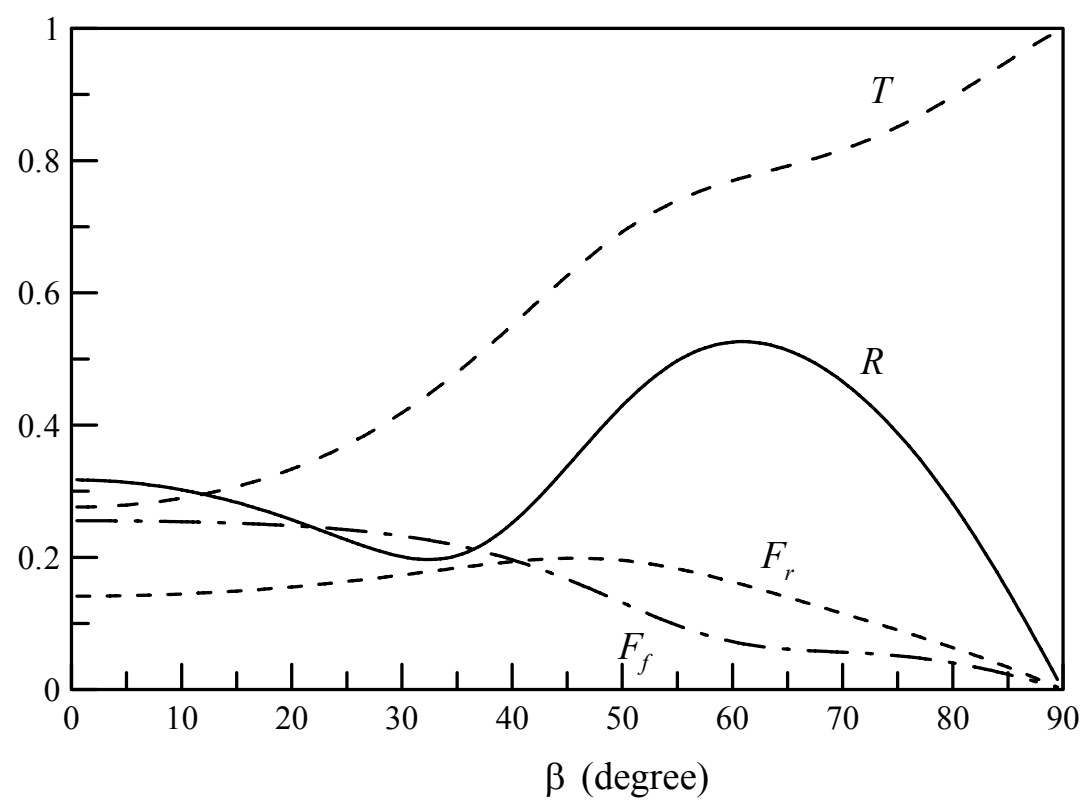

Figure 7. Effects of $\beta$ on $R, T, F_{f}$ and $F_{r}: k_{0} d=2.0, G=0.3, a / d=0.4$ and $B / L=0.2$. 


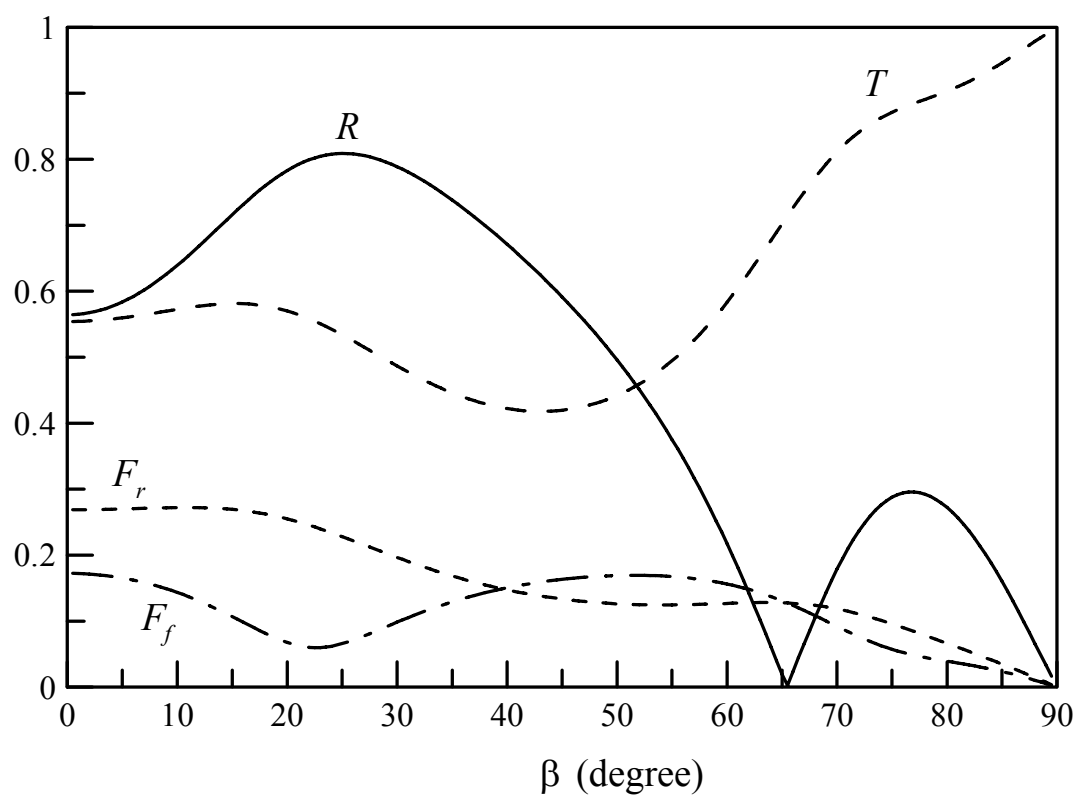

Figure 8. Effects of $\beta$ on $R, T, F_{f}$ and $F_{r}: k_{0} d=2.0, G=0.3, a / d=0.4$ and $B / L=0.6$.

Fig. 9 shows the effects of the relative draft $a / d$ on $R, T, F_{f}$ and $F_{r}$ at $k_{0} d=2.0, G=0.3, B / L=0.2$ and $\beta=30^{\circ}$. It is evident from Fig. 9 that with the increasing value of the relative draft, the transmission coefficient decreases to zero monotonously. However, the reflection coefficient does not necessarily increases with the increasing value of $a / d$. This has also been observed in the experimental tests of Brossard et al. (2003). This phenomenon should be carefully considered in practical design of partially immersed wave absorbing breakwaters. From Fig. 9, we also note that the dimensionless wave force on the front barrier increases with the increasing value of ald. But the dimensionless wave force on the rear barrier decreases slightly when a/d exceeds 0.4 .

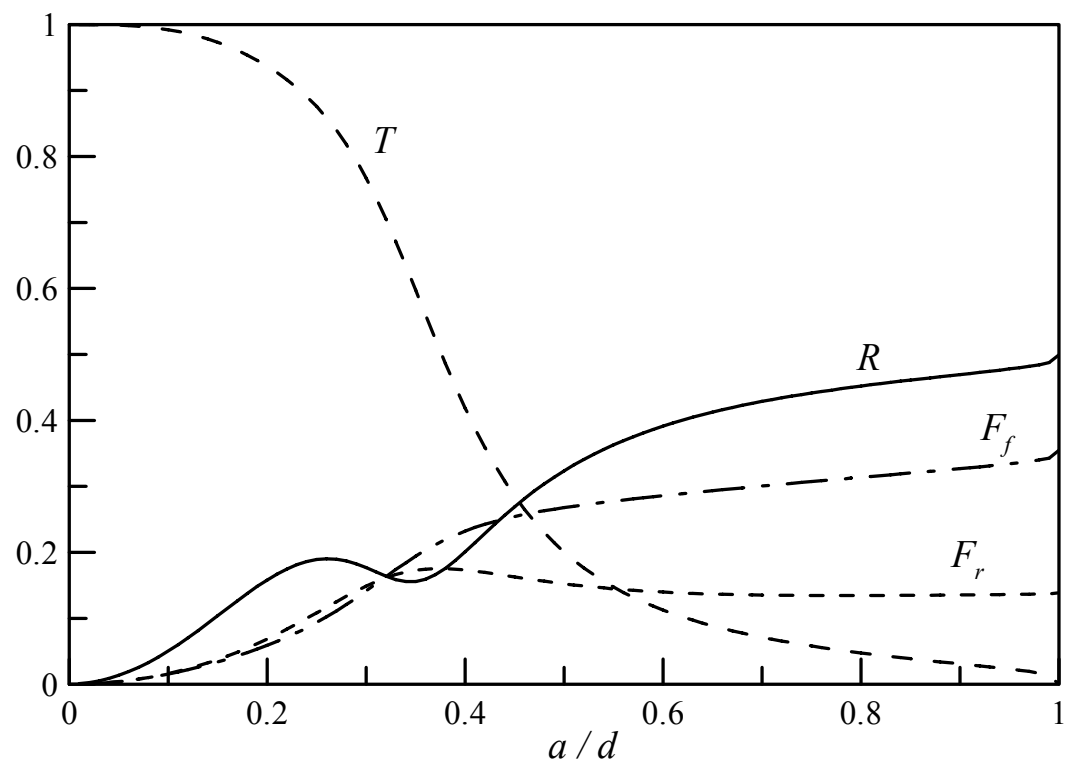

Figure 9. Effects of ald on $R, T, F_{f}$ and $F_{r}: k_{0} d=2.0, G=0.3, B / L=0.2$ and $\beta=30^{\circ}$. 
Fig. 10 shows the effects of $|G|$ on $R, T, F_{f}$ and $F_{r}$ at $k_{0} d=2.0, G=|G| \mathrm{e}^{0 \mathrm{i}}, B / L=0.2, a / h=0.4$ and $\beta=30^{\circ}$. It is evident from this figure that the reflection and transmission coefficients attain minimum at a moderate value of $G$ (a moderate geometrical porosity of the perforated barrier). At this time, the energy loss coefficient calculated by $1-R^{2}-T^{2}$ attains maximum. This has been observed by $\mathrm{Yu}$ and Chwang (1994) for a submerged horizontal porous plate. From Fig. 10, it is also evident that the dimensionless wave force on the perforated front barrier decreases to zero with the increasing value of $|G|$. This is physically natural. But the variation of $|G|$ has no significant effect on the dimensionless wave force on the rear barrier.

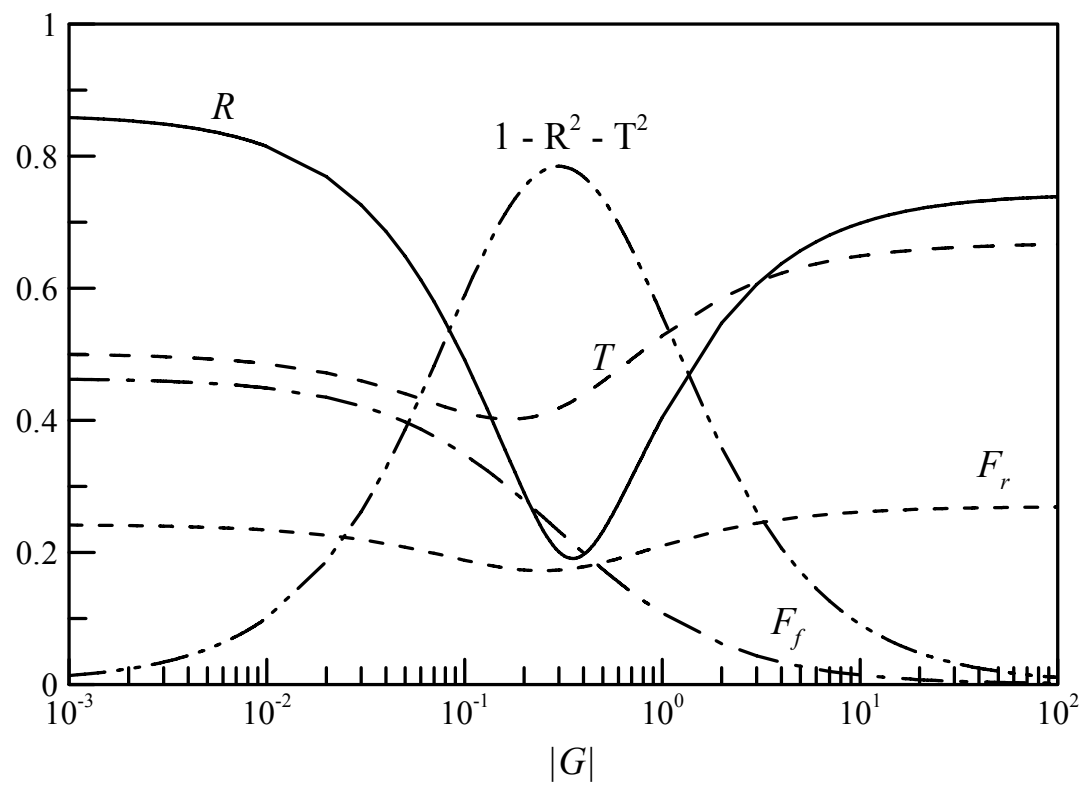

Figure 10. Effects of $|G|$ on $R, T, F_{f}$ and $F_{r}: k_{0} d=2.0, G=|G| e^{0 i}, B / L=0.2, a / h=0.4$ and $\beta=30^{\circ}$.

According to the above numerical results, the hydrodynamic performance of the partially immersed wave absorbing breakwater is sensitive to the variations of $B / L, \beta$, ald and $G$. So theses parameters must be carefully determined in practice design. Also we note that the inertial effect of the perforated barrier (denote by the imaginary part of $G$ ) is not analyzed. Generally, the inertial effect may lead to lower reflection coefficients at smaller values of $B / L$. For a very thin perforated barrier, the inertial effect may be not significant.

\section{CONCLUSIONS}

This study has developed an analytical solution to the scattering of obliquely incident linear water waves by a partially immersed wave absorbing breakwater consisting of a perforated front barrier and a solid rear barrier. The eigenfunction expansion method and a least square method are adopted in the solution. The present solution has been validated by comparing calculated results with previous predictions for limiting cases and previous experimental data. Numerical examples have been presented to examine the hydrodynamic performance of the breakwater. It is found that the wave absorbing performance of the breakwater is sensitive to the relative space between the two barriers, the angle of incident waves, the relative draft of the breakwater and the porosity of the front barrier. With suitable relative space between two barriers, the reflection coefficient, the transmission coefficient and the dimensionless wave force on each barrier may be simultaneously small. A moderate angle of incident waves is more helpful to obtain better wave absorbing performance and smaller wave forces. Increasing the relative draft decreases the transmission coefficient, but does not necessarily increase the reflection coefficient. The new developed solution may be used at a preliminary design stage for practical designs. Also the present results should be useful to the further experimental investigations. 


\section{ACKNOWLEDGEMENTS}

This work was financially sponsored by the Foundation for Innovative Research Groups of the National Natural Science Foundation of China (50921001), the National Natural Science Foundation of China (50909086, 50609001), and the Natural Science Foundation of Shandong Province (Q2008F01).

\section{REFERENCES}

Bennett, G.S., McIver, P., and Smallman, J.V., 1992. A mathematical model of a slotted wavescreen breakwater. Coastal Engineering, 18, 231-249.

Brossard, J., Jarno-Druaux, A., Marin, F., and Tabet-Aoul, E.H., 2003. Fixed absorbing semiimmersed breakwater. Coastal Engineering, 49, 25-41.

Chwang, A.T., and Dong, Z., 1984. Wave-trapping due to a porous plate. Proceedings of the $15^{\text {th }}$ Symposium on Naval Hydrodynamics, National Academy Press, Washington, D.C., pp. 407-417.

Cox, R.J., Horton, P.R., and Bettington, S.H., 1998. Double walled, low reflection wave barriers. Proceedings of the $26^{\text {th }}$ Coastal Engineering Conference, ASCE, Copenhagen, Denmark, pp. 2221-2234.

Dalrymple, R.A., and Martin, P.A., 1990. Wave diffraction through offshore breakwaters. Journal of Waterway, Port, Coastal, and Ocean Engineering, ASCE 116 (6), 727-741.

Das, P., Dolai, D.P., and Mandal, B.N., 1997. Oblique wave diffraction by parallel thin vertical barriers with gaps. Journal of Waterway, Port, Coastal, and Ocean Engineering, ASCE 123 (4), $163-171$.

Fugazza, M., and Natale, L., 1992. Hydraulic design of perforated breakwaters. Journal of Waterway, Port, Coastal, and Ocean Engineering, 118 (1), 1-14.

Jarlan, G.E., 1961. A perforated vertical wall breakwater. The Dock and Harbour Authority, 41 (486), 394-398.

Li, Y.C., 2007. Interaction between waves and perforated-caisson breakwaters. Proceedings of the 4th International Conference on Asian and Pacific Coasts 2007, Nanjing, China, pp. 1-16.

Li, Y.C., Liu, Y., and Teng, B., 2006. Porous effect parameter of thin permeable plates. Coastal Engineering Journal 48 (4), 309-336.

Porter, R., 1995. Complementary methods and bounds in linear water waves. Doctoral thesis, University of Bristol.

Sollitt, C.K., and Cross, R.H., 1972. Wave transmission through permeable breakwaters. Proceedings of the $13^{\text {th }}$ Coastal Engineering Conference, Vancouver, pp. 1827-1846.

Suh, K.D., Park, J.K., Park, W.S., 2006. Wave reflection from partially perforated-wall caisson breakwater. Ocean Engineering, 33, 264-280.

Tanimoto, K., Yoshimoto, Y., 1982. Theoretical and experimental study of reflection coefficient for wave dissipating caisson with a permeable front wall. Report of the Port and Harbour Research Institute, 21 (3), 44-77 (in Japanese, with English abstract).

Yu, X.P., 1995. Diffraction of water waves by porous breakwaters. Journal of Waterway, Port, Coastal, and Ocean Engineering, 121 (6), 275-282.

Yu, X.P., Chwang, A.T., 1994. Water waves above submerged porous plate. Journal of Engineering Mechanics, 120 (6), 1270-1282. 